\title{
Evolución y perspectivas de la población francesa en las últimas décadas 1
}

\author{
Yvanne Bouvet
}

Laboratoire Géoarchitecture, Territoire Urbanisation, Biodiversité, Environnement Université de Bretagne occidentale, France I yvanne.bouvet@univ-brest.fr

Este texto corresponde a la conferencia dictada durante las XVIII Jornadas de Investigación organizadas por el Departamento de Geografía y el Centro de Investigaciones Geográficas de la Universidad Nacional de La Plata, realizadas en el mes de noviembre de 2016 en la Facultad de Humanidades y Ciencias de la Educación de dicha Universidad. Se trata de un resumen que combina temas desarrollados en los cursos de Geografía de Francia y Geografía de la Población, que se dictan en la Universidad de Bretaña Occidental -Sede Brest-.

La población francesa es resultado de una mezcla de aquellos pueblos que conformaron el gran conjunto europeo. Francia fue hasta el siglo XVIII el país más poblado de Europa, pero desde entonces ha conocido una realidad diferente de la de sus vecinos. Su transición demográfica fue precoz, anticipándose a menudo a las situaciones de su tiempo.

Esta presentación se organiza en tres partes: para comprender cómo se produjo la distribución de la población francesa en el territoriodebemos ver la evolución demográfica; después nos ocuparemos de la composición por edades, que hace de Francia un país viejo; pero, por suerte, es también una tierra de inmigrantes por lo que, al final, nos introduciremos en el problema de los refugiados.

\section{Evolución demográfica}

Las informaciones sobre la población de Francia son dudosas hasta el primer censo de población, realizado en 1801. Sabemos que Francia fue un lugar poblado de modo precoz y tiene más muertos que vivos en su suelo; al final del paleolítico contaba con cincuenta mil habitantes (Figuras1 y 2). 
Figura 1 - Evolución de la población francesa a partir de la Galia romana

(para el territorio actual, en millones de habitantes)

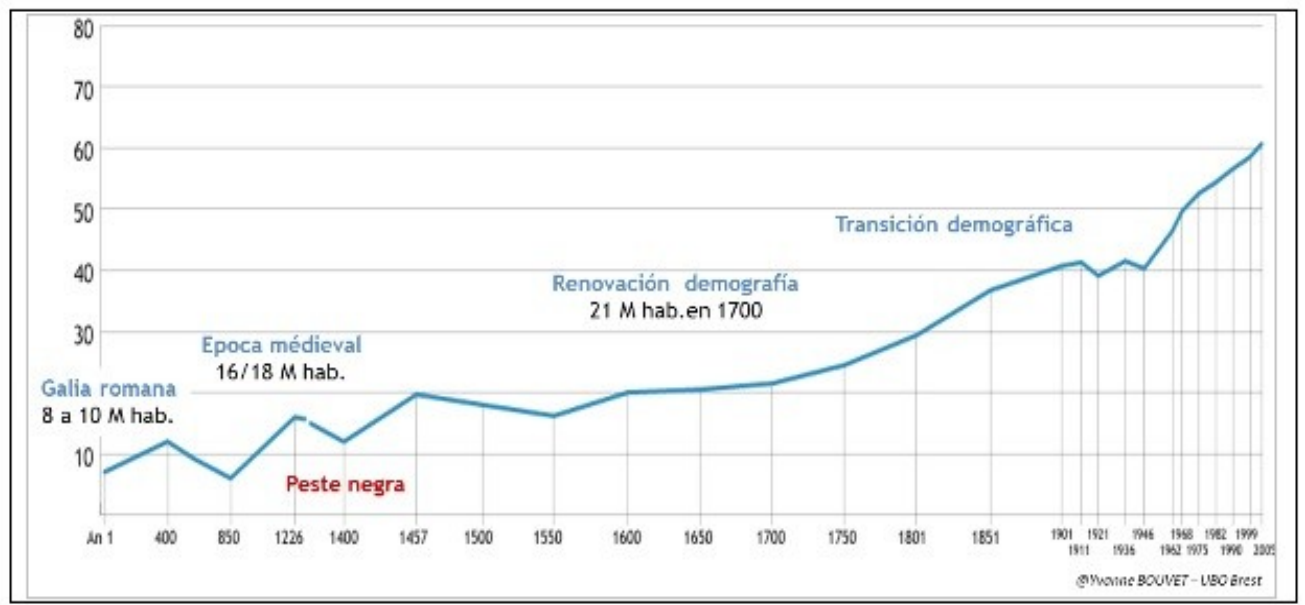

Fuentes: INSEE, $2010^{2}$.

En el neolítico, entre tres mil y mil quinientos años antes de Cristo, la población pasó de uno a cuatro millones de habitantes. Al principio de la era cristiana, la Galia romana tenía alrededor de 7 millones de habitantes, el primer crecimiento se produjo en la época medieval, durante el siglo XII, cuando la población era de casi 20 millones, pero la mitad de la misma sería diezmada por la peste negra y las guerras de los siglos XIII y XIV. El período entre los siglos XV y XVIII vio aumentar lentamente la población hasta alcanzar los 30 millones de habitantes en el año 1801, año del primer censo de la República, con una particularidad: un siglo antes que sus vecinos podemos ver una disminución de la natalidad mientras que la mortalidad se mantenía alta. El siglo XIX vio pasar la población de 30 a 40 millones de habitantes, con un fuerte crecimiento durante la primera mitad del siglo y una disminuciónen la segunda. El crecimiento natural se vio atenuado por una fecundidad baja, asociada a una mortalidad alta a pesar de los progresos médicos. Este crecimiento del siglo XIX se explica por la acogida de emigrantes (1,2 millones en un siglo) que llegaban de países europeos vecinos (Bélgica, Italia, Polonia) para sustentar la revolución industrial, y también para compensar las emigraciones de los franceses (2,4 millones entre 1830 y 1917), principalmente hijos de campesinos pobres con destino a colonias francesas como Argelia. Pero una gran parte de estos emigrados también se dirigió a América, a los Estados unidos, Argentina, Uruguay, etc. En esta época Francia inició una política de naturalización de los extranjeros, con la incorporaciónal Código Civil del derecho del suelo (un niño nacido en Francia es francés). 
Figura 2 - Evolución de la población francesa de 1901 a 2014

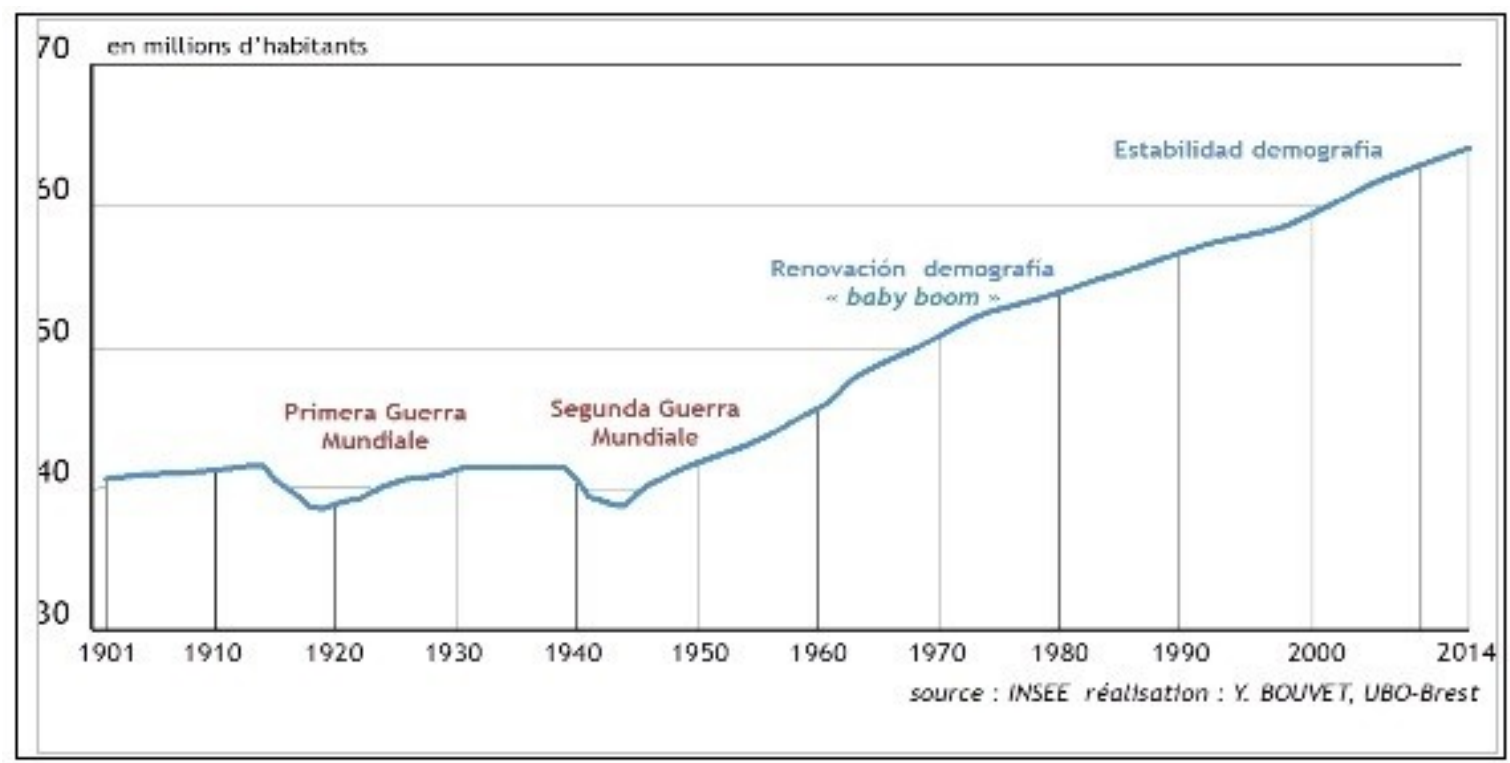

El siglo XX fue el siglo de las guerras mundiales sobre todo en Europa. La primera guerra dejó 1,4 millones de muertos, es decir 3,4\% de la población.

Fueron hombres jóvenes los que murieron: un hombre cada 5 nacidos entre 1887 y 1895, y un hombre cada 3 nacidos en el año 1894 (que tenían 20 años en 1914). La natalidad bajó bruscamente (figura 3), los hombres estaban en guerra, había muchas viudas y no se producían matrimonios. El costo demográfico de este conflicto fue de 3 millones de personas; es la suma de los muertos y de los niños no-nacidos, dentro de las clases de jóvenes adultos y de niños de menos 5 años. Esta sangría de la primera guerra mundial no será saldada hasta el principio del segundo conflicto.

La segunda guerra produjo menos muertes (580 mil), pero la caída fue un poco mayor al separarse las familias.Al final de la guerra había 40 millones de franceses, es decir dos millones menos que en 1913. Durante los años siguientes, de 1942 a 1972, se renovaron las condiciones demográficas; con un nivel alto de natalidad, una inmigración importante y una disminución de la mortalidad, la población creció ganando 10 millones de personas en 30 años. Este es el fenómeno del baby boom, de un mejoramiento de la economía, de políticas públicas que apoyaban a las familias y a los niños (escuelas gratis para todos, prestaciones familiares) y con la prioridad puesta en una salud para todos. 
Figura 3 - Natalidad y mortalidad de la población francesa de 1901 a 2014

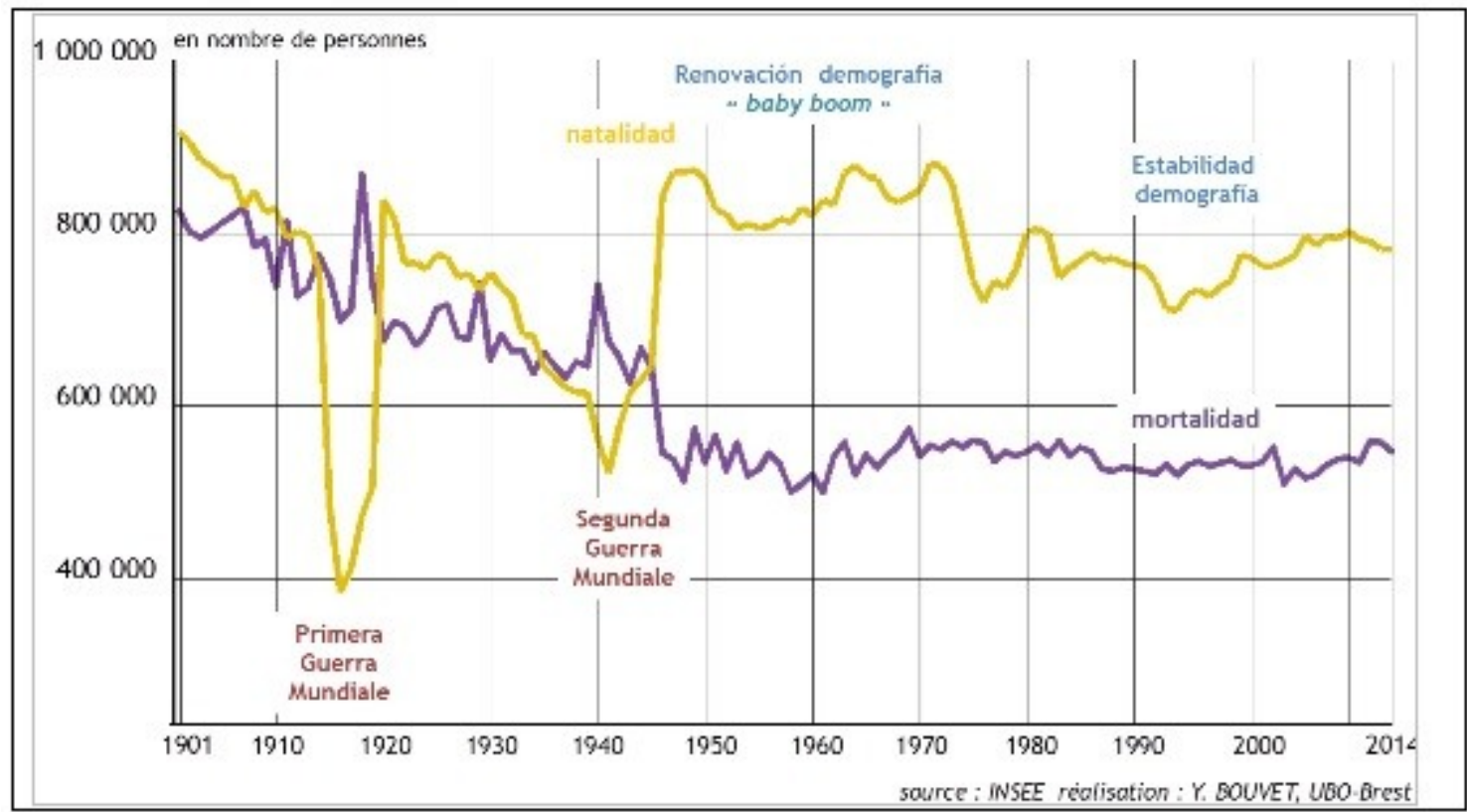

Figura 4 - Crecimiento natural de la población francesa de 1901 a 2014

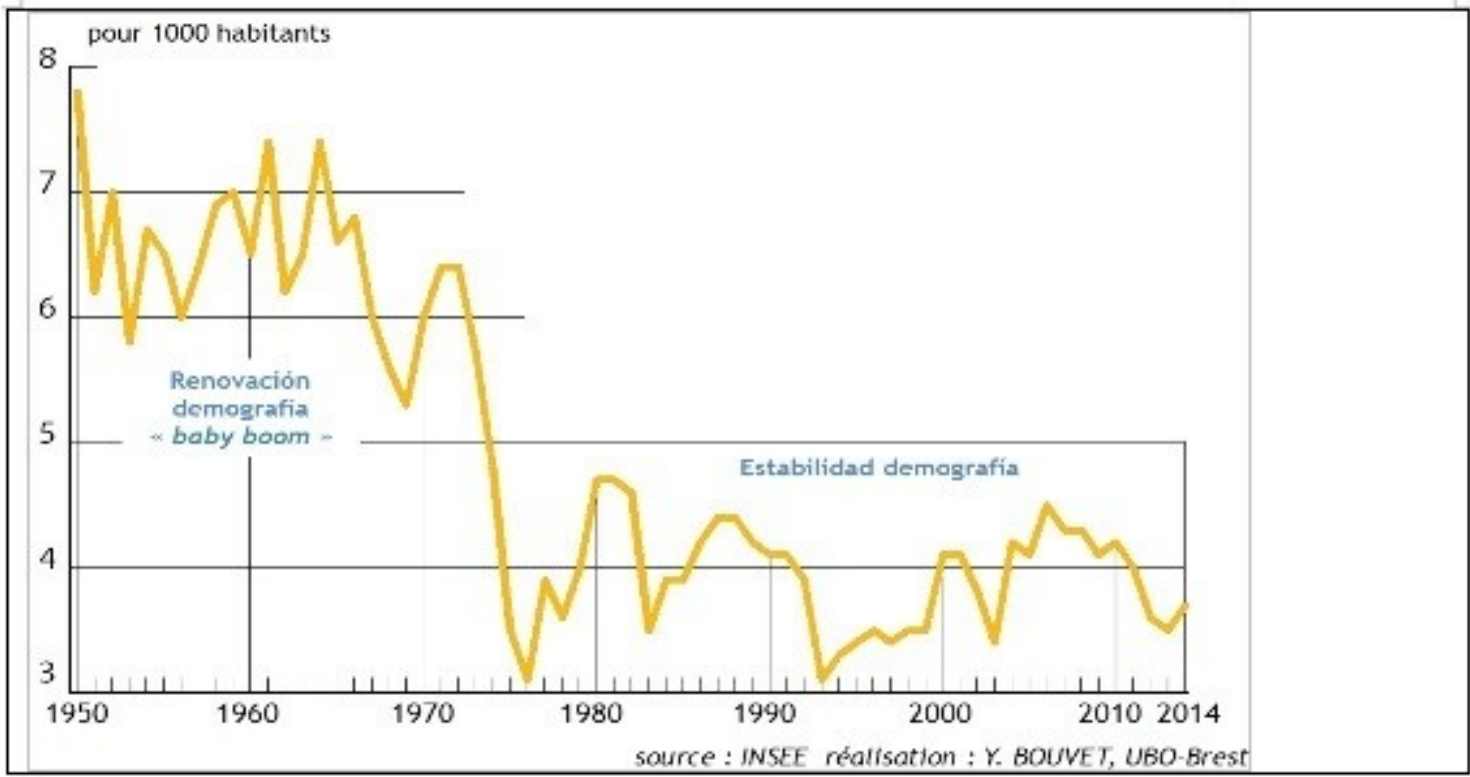

El último cuarto del siglo XX y el inicio de los años 2000 vieron la estabilización del crecimiento natural de la población en un 3,3 por mil en 2012, con una natalidad de 12,5 por mil y una mortalidad de 8,8 por mil (figura 4). Pese a una mortalidad infantil estabilizada en 3,3 por mil, la mortalidad decrece, la población envejece y no sabemos cómo va ser la natalidad. Francia tiene un comportamiento diferente de sus vecinos, con una tasa de fecundidad alta, 2 niños por mujer a pesar de la crisis económica o del desempleo.

Sin embargo, con un balance demográfico positivo, la población francesa ocupa una parte de menor importancia dentro de la población mundial. A mediados del siglo XVIII Francia representaba el 15\% de la población europea y el 3\% de la población mundial. Hoy, representa menos del 8\% de los europeos y menos del $1 \%$ de los habitantes de la Tierra. 


\section{La composición de la población}

Francia es un país que envejece, y se presentacomo un país viejo. La proporción de personas mayores crece al contrario de lo que ocurre con las personas jóvenes.

El sector de las personas de 65 años y más era de 11,4\% a mediados del siglo XX; es de 19\% en 2015 (figura 5). El sector de las personas menores de 20 años representaba 30\% en 1950 y 25\% después del inicio de los años 2000.

Figura 5 - Pirámide de edades de Francia en 2015

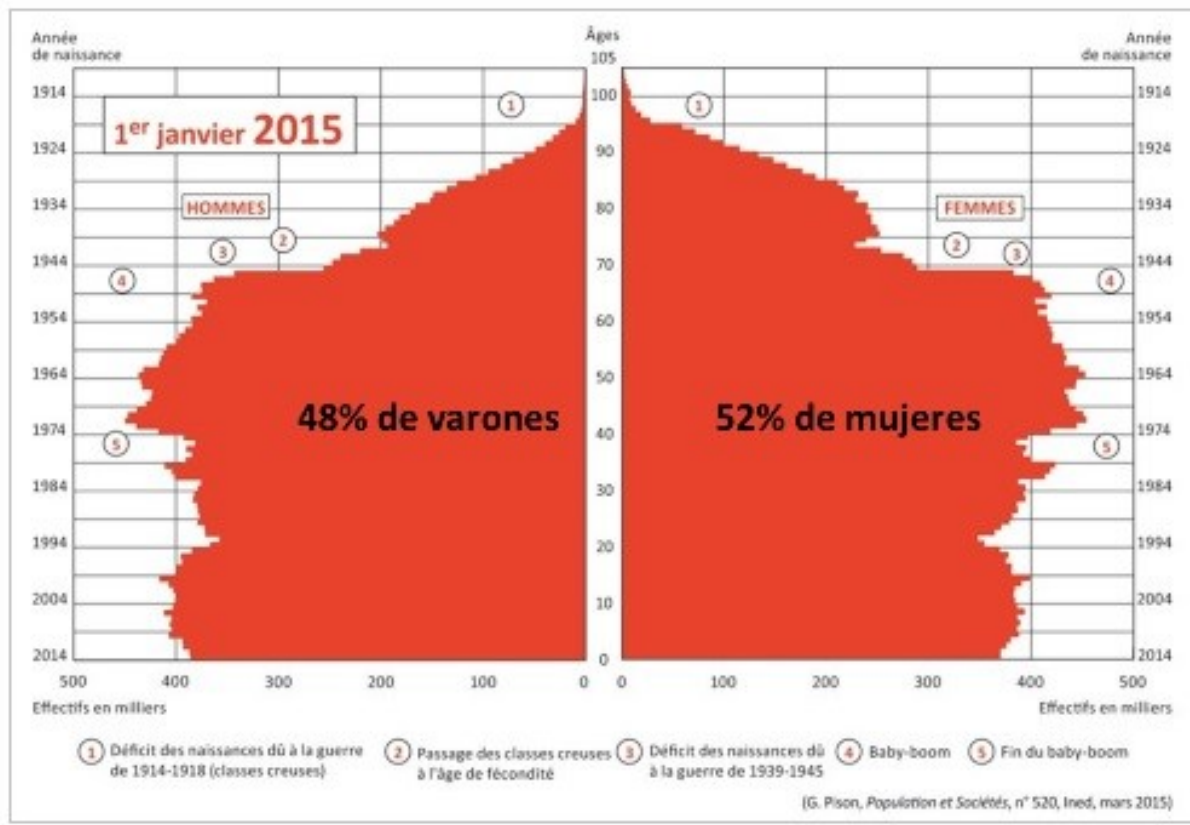

La estructura por edades es característica de una población que envejece, es el reflejo de la historia demográfica del siglo pasado. La cima es muy asimétrica como consecuencia de que las mujeres viven más (esperanza de vida: 85 años) que los hombres (78,7 años) y, en número, las mujeres son 2, 3, 4 o más veces que los hombres según las clases de edades. La muesca simétrica de la primera guerra mundial se ve cada vez menos, pero se distingueque la llegada de la clase a la edad de procreación en 1935 es más baja (es el déficit de nacimiento de la gente no-nacida que no tienehijos), lo que está asociado al déficit de nacimientos de la segunda guerra mundial (menos fuerte que en la primera). La ampliación de la pirámide para la gente que tiene de 40 a 70 años de edad es la consecuencia del baby boom (de 1942 a 1972), seguida después por sectores menos numerosos. Podemos ver la fluctuación de la natalidad con un nivel más fuerte en el inicio de los años 1980 y 2000.

Por medio de la pirámide de edades sabemos que la evolución natural va a disminuir, porque el número de mujeres que pueden tener hijos es estable y el número de viejos crece -y con ellos el crecimiento de la mortalidad-. Para los futuros 30 años la inmigración será la única posibilidad de ver la población crecer.

Hasta mediados del siglo XVIII, la población francesa vivía sobre todo en el campo, excepto el caso de París y de algunas ciudades de provincia (como Lyón, Marsella). Con la revolución industrial, la población empieza a trabajar en fábricas y a concentrase cerca de los sitios de producción. Los siglos XIX y XX son sinónimos de éxodo rural, con un aumento de la población,la mecanización de la agricultura y los campesinos convertidos en obreros industriales urbanos. Pero debemos esperar hasta 1930 para ver que el índice de urbanización sea del 50\%, un siglo más tarde que en Inglaterra y 60 años detrás de Alemania. Hoy, el índice de urbanización es del 78\% de la población, con un crecimiento lento. 
Figura 6 - Densidad de la población francesa en 2009 (habitante por $\mathrm{km}^{2}$ )

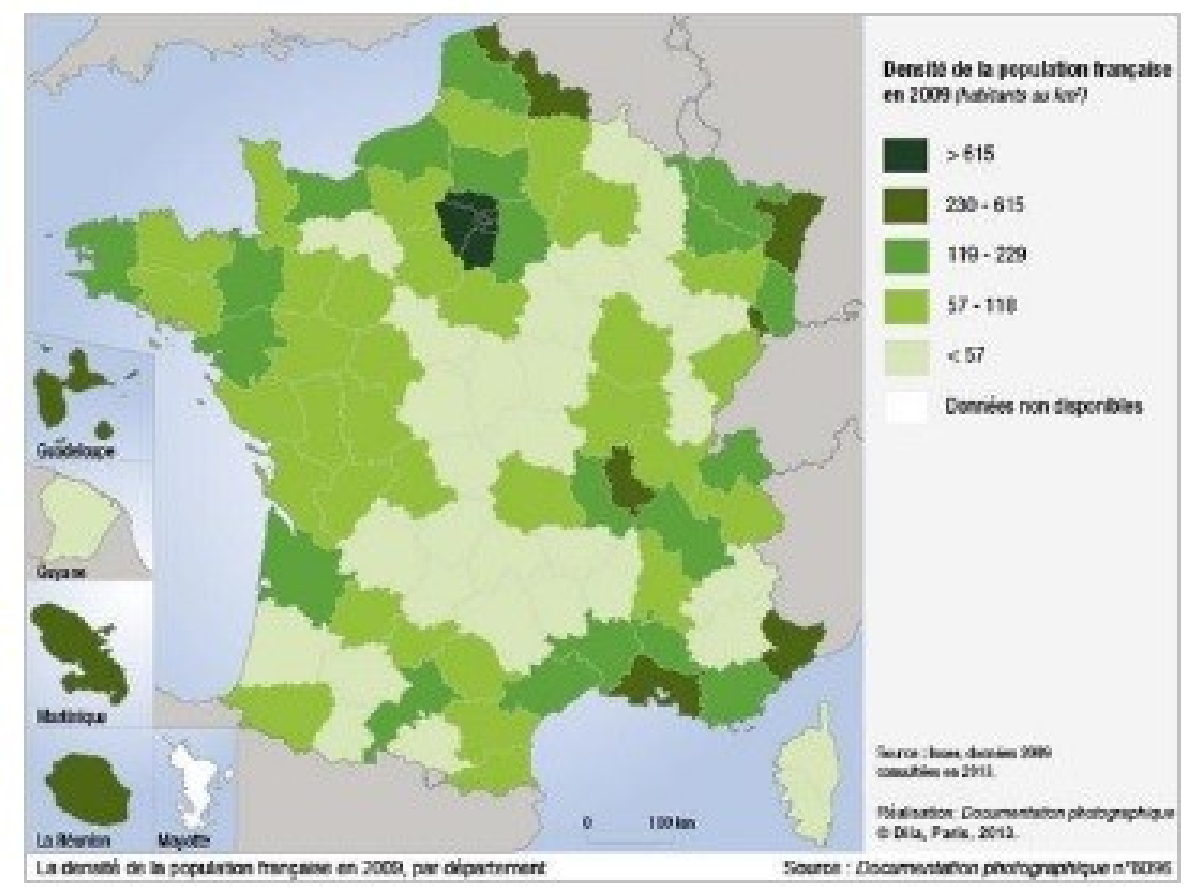

El reparto de la población se puede ver en el mapa de la densidad de población (figura 6) que muestra una media de $114 \mathrm{hab} / \mathrm{km}^{2}$, pero con menos de $57 \mathrm{hab} / \mathrm{km}^{2}$ en el centro de Franciay más de $600 \mathrm{hab} / \mathrm{km}^{2}$ en el centro de las ciudades. La concentración de población puede verse en las ciudades, en primer lugar está París y su área metropolitana (11 millones de habitantes sobre 62 para toda Francia), y las grandes ciudades de más de 1 millón de habitantes (Lila, Estrasburgo, Lyón, Marsella, Niza, Toulouse). Vemos también las zonas industriales del norte y este de Francia, cerca de las fronteras con Bélgica, Alemania, Suiza, así como la concentración alrededor de los ríos Ródano y Saona, o del Sena desde el puerto de ElHavrehasta París. Pero hay un fenómeno reciente de instalación de población (especialmente de jubilados) en el oeste y sur del país, en las provincias delacostadel Atlántico y sobre el litoral del Mediterráneo. En estos lugares hay ciudades con ríos y puertos marítimos (Burdeos, Nantes, Toulouse, Ruán/El Havre) y también nuevas actividades de ocio y turismo.

Hay otras zonas que conforman la "Francia del vacío", con menos cantidad de habitantes, poblaciones viejas, menos actividades económicas (agricultura de montaña: Alpes, Pirineos, Macizo central, Ardenas). Es la Francia rural de donde los jóvenes se fueron para ir a trabajar a las ciudades. 
Figura 7 - El sistema urbano francés

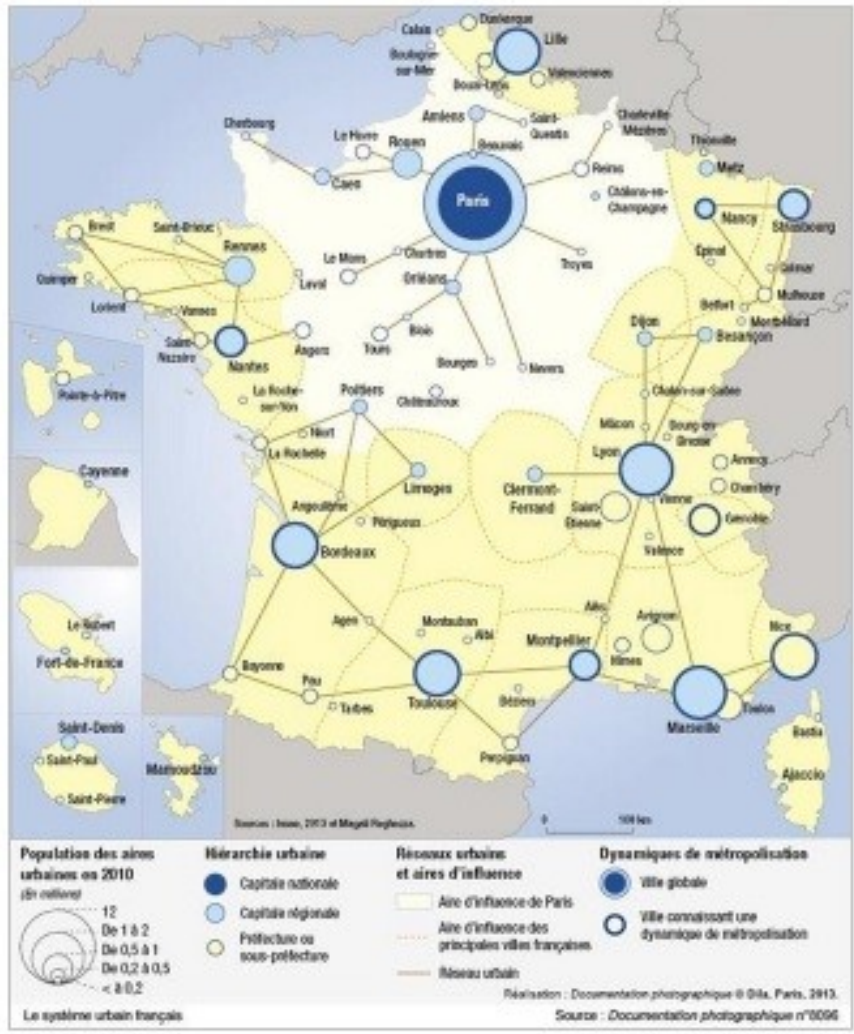

La distribución de la población se puede ver sobre una red de ciudades (de tipo de Christaller) que organiza los espacios, con fuerte predominio de algunas “metrópolis regionales”, como se ve en el mapa del Figura 7.

Figura 8 - Evolución de la población francesa de 1990 a 2007 


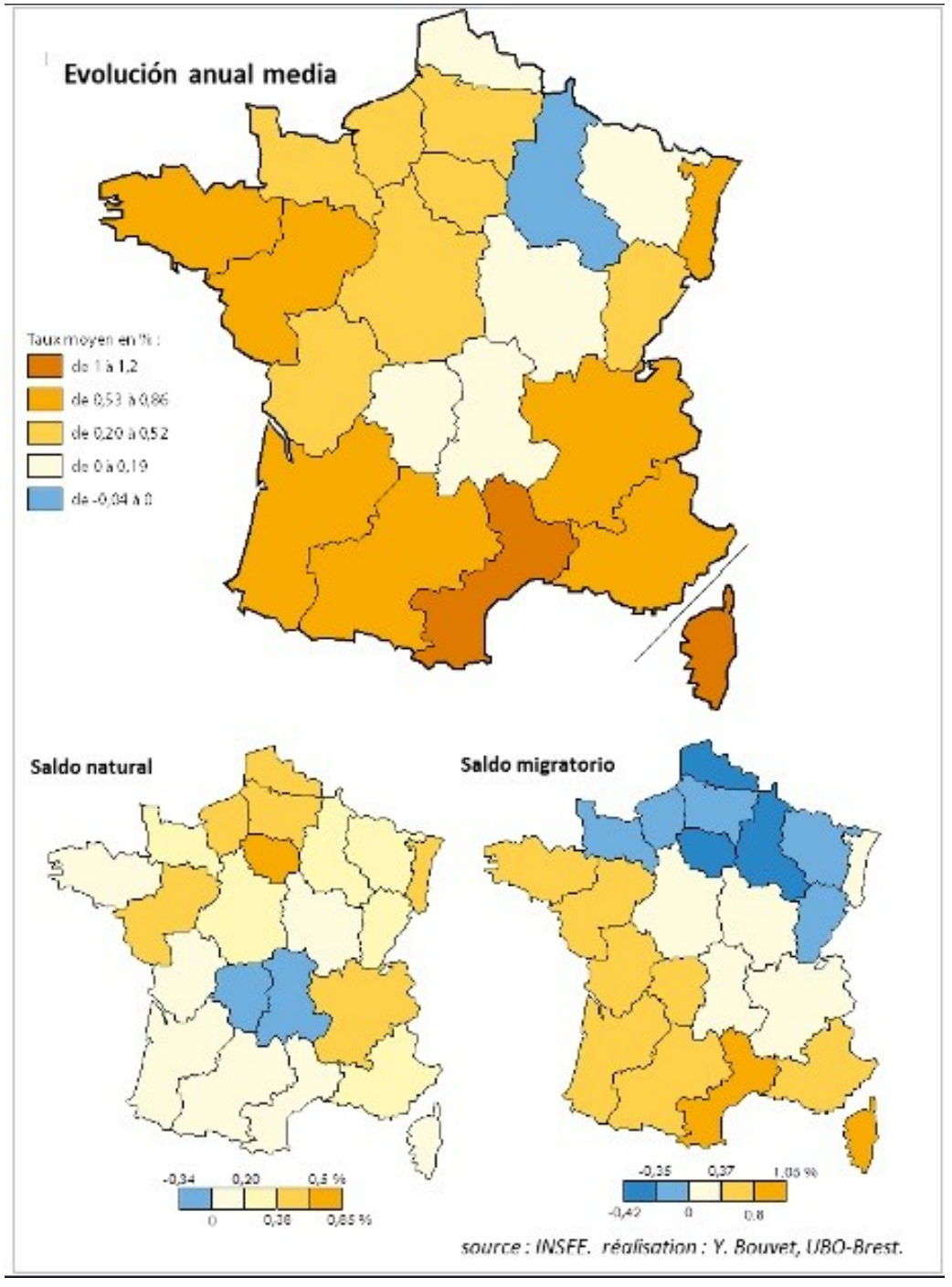

Con los mapas de evolución anual media en casi 30 años (Figura 8) podemos ver el contraste entre una Francia dinámica del sur y del oeste y una Francia interior que ve disminuir su población. Con las informaciones sobre saldos naturales y de migración vemos una población con dinámica natural en Paris, en el norte, en el este (Alsacia con Estrasburgo, y alrededores de Lyon); hay una nueva zona dinámica en el oeste, Nantes. El centro de macizos y montañas de Francia pierde población porque es vieja. Al contrario, es la Francia del litoral a donde va la población para vivir. Este fenómeno se llama la Francia invertida, porque vemos invertirse regiones dinámicas demográfica y económicamente hablando. La población de las antiguas regiones industriales y urbanas del norte y este de Francia va a las regiones litorales que tienen actividades nuevas, como servicios, turismo, investigaciones científicas y nuevas tecnologías industriales.

Es el resultado de una política de equilibrio del espacio y de descentralización del poder político y económico, desde los años 1960, para disminuir el poder de París. Vemos aparecer unidades urbanas de quinientos mil habitantes a dos millones lejos de la capital que tienen más población y actividades económicas.

\section{Francia, tierra de inmigrantes}

Francia es un país de datos.Desde Napoleón, al inicio del siglo XIX, hasta el 2007, tenemos datos sobre la población con censos que se hacían siempre (o casi siempre) cada cinco años, si no había guerras, y también contamos con el Registro Civil con muchas informaciones sobre nacimientos, muertes, matrimonios. De la misma manera, tenemos datos sobre las poblaciones migratorias legales: extranjeros que llegan a Francia o 
franceses que se van a otros países. Así, si bien en Francia no hay datos sobre el carácter étnico, hay información sobre lo que se llama los immigrés, inmigrantes nacidos en otro país o de padres extranjeros y que viven en Francia. Algunos pueden ser franceses por naturalización (figura 9).

\section{Figura 9 - Población francesa y parte de inmigrantes después del medio del siglo 19}

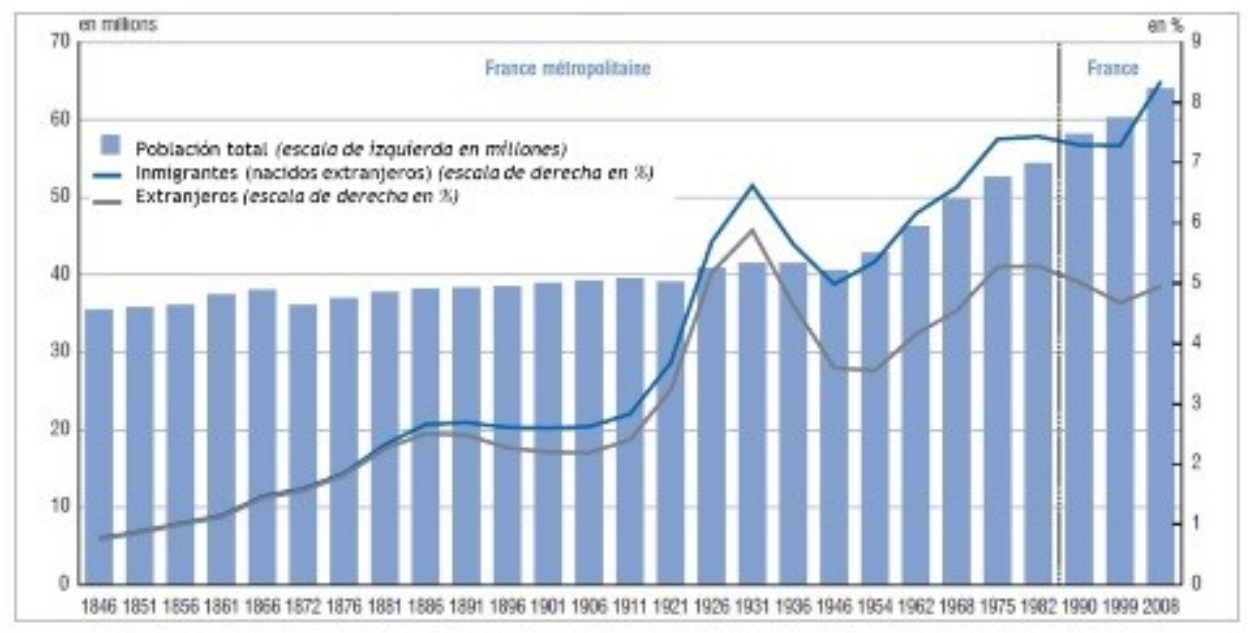

Fuentes: INSEE, 2010

La población que vivía en Francia en 2014 era de 66,3 millones de habitantes. Se componía de:

- Población francesa nacida en Francia: 57,6 millones de habitantes

- Inmigrantes que tienen nacionalidad francesa: 2,3 millones de habitantes (3\% de la población total)

- Los franceses nacidos fuera de Francia: 1,7 millones de habitantes.

- Población extranjera que vive en Francia : 4,2 millones de habitantes (6\% de la población total), con 0,6 millones de habitantes nacidos en Francia

La suma de la población inmigrante en 2014 era de 6,5 millones de habitantes, es decir, 9 \% de la población total.

El problema es que mucha gente es identificada con la palabra inmigrantes, también los hijos de los inmigrantes verdaderos, o los extranjeros, naturalizados o no, lo que hace un 9\% de la población.

Desde el siglo XIX, con las revoluciones industriales y la necesidad de mano de obra, los inmigrantes han venido a vivir y a trabajar en Francia, integrándose a la población francesa y haciéndose ciudadanos franceses. Se dice en Francia que todo francés tiene un abuelo sobre 4 que era inmigrante. Por ejemplo, Nicolas Sarkozy (que fue Presidente de Francia) proviene de Grecia y Hungría por sus abuelos, y Manuel Valls (que quiere ser presidente) fue naturalizado a los 20 años, con padre español y madre suiza.

Después del crecimiento por la llegada de extranjeros luegode la primera y la segunda guerras mundiales, el número de extranjeros se ha estabilizado-desde los años 1980- con un pequeño crecimiento en los últimos años (alrededor de 330.000 personas llegan por año al espacio francés, y 5 de cada 10 vienen de Europa: Portugal, Gran Bretaña, España, Italia, Alemania, 3 de África: Marruecos, Túnez, Argelia). Desde hace algunos años son más las mujeres jóvenes (54\%) que llegan a Francia (estudiantes, familiares, matrimonios)que los hombres. Los nuevos inmigrantes logran títulos, 6 cada 10 tienen el 
baccalaureat(primer diploma de la Universidad).

La primera ola de migración se ubica en la segunda mitad del siglo XIX, con inmigrantes que vienen de Bélgica, de Italia y de España. Entre las dos guerras mundiales están los inmigrantes de Italia, de Polonia, de España, loscuales van a decrecer después de la segunda guerra. Con la reconstrucción y los "Treinta gloriosos” y la descolonización llegan migraciones del Magreb (Argelia, Marruecos, Túnez) y más tarde de otros países de África y de Turquía. Son principalmenteagrupamientos familiares, migraciones de estudiantes y otros que llegan un poco por razones económicas o para protegerse de las guerras.

Los inmigrantes viven en ciudades (donde hay industrias y servicios) como se ve en el mapa dela figura 10; en la región de París, en las regiones cerca de las fronteras del noreste, y en zonas agrícolas, en el sur de Francia, cerca del Mediterráneo (con empleos en servicios del turismo), en el suroeste, donde se necesita mano de obra para la recolección de frutas.

El oeste de Francia no tiene muchos inmigrantes excepto en el centro de Bretaña, pero son europeos que aprovechan precios inmobiliarios baratos en el espacio rural muy profundo.

Hay también franceses que viven en otros países del mundo (en casi todos los países); son 1,6 millones, número que aumentó en un 100\% después de 1995. Esta evolución se explica por el aumento de los movimientos entre los países europeos (migraciones de estudiantes con el programa ERASMUS, personas que trabajan en firmas transnacionales), lo que explica, en parte, que las migraciones más importantes están en Europa, en Norteamérica, y alrededor del Mediterráneo.

\section{Figura 10 - Los inmigrantes en la población total de Francia en 2014}

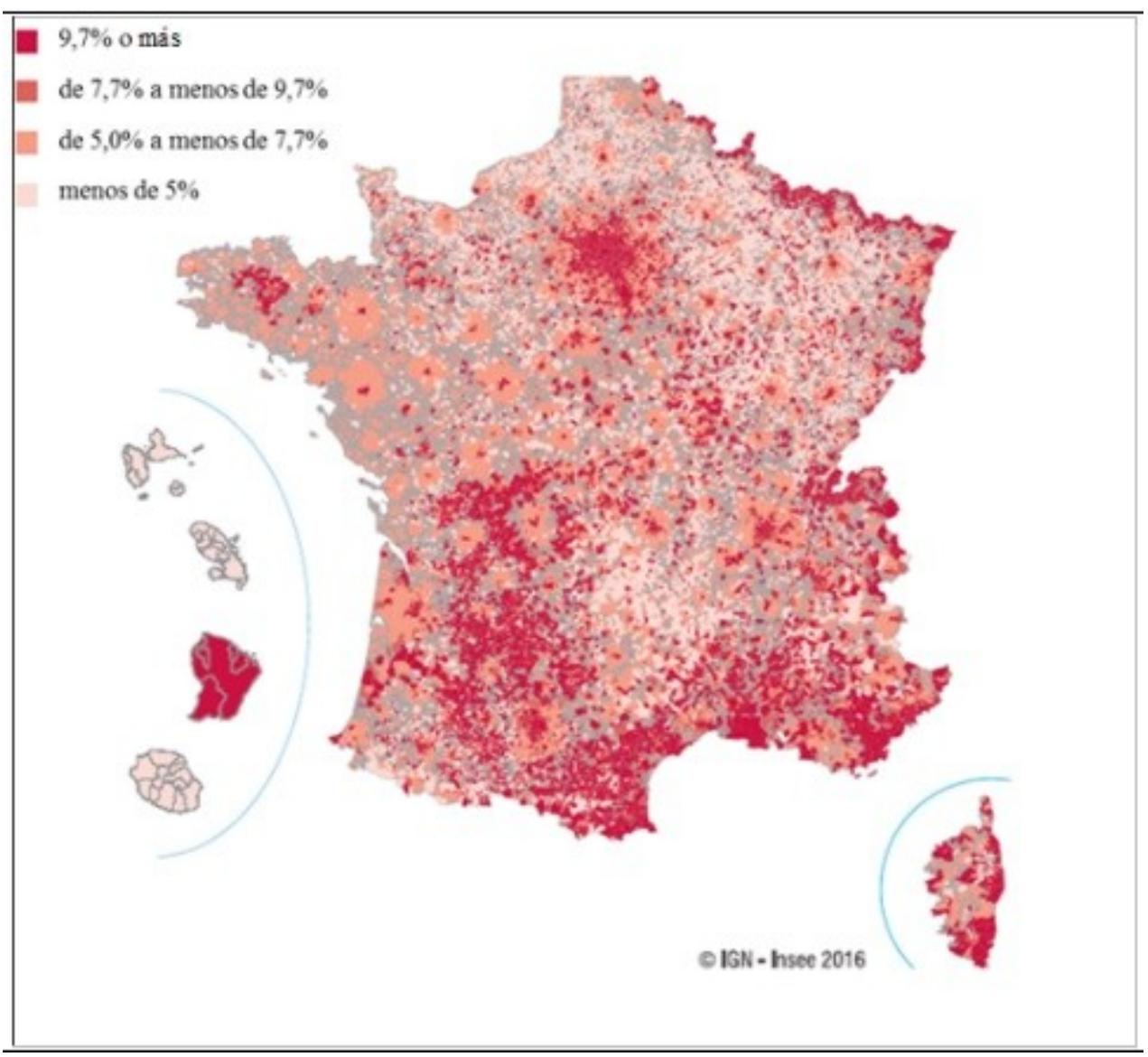




\section{Europa, inmigrantes y refugiados}

\section{Figura 11 - Evolución de tasas de natalidad y mortalidad en Europa de 1961 a 2014}

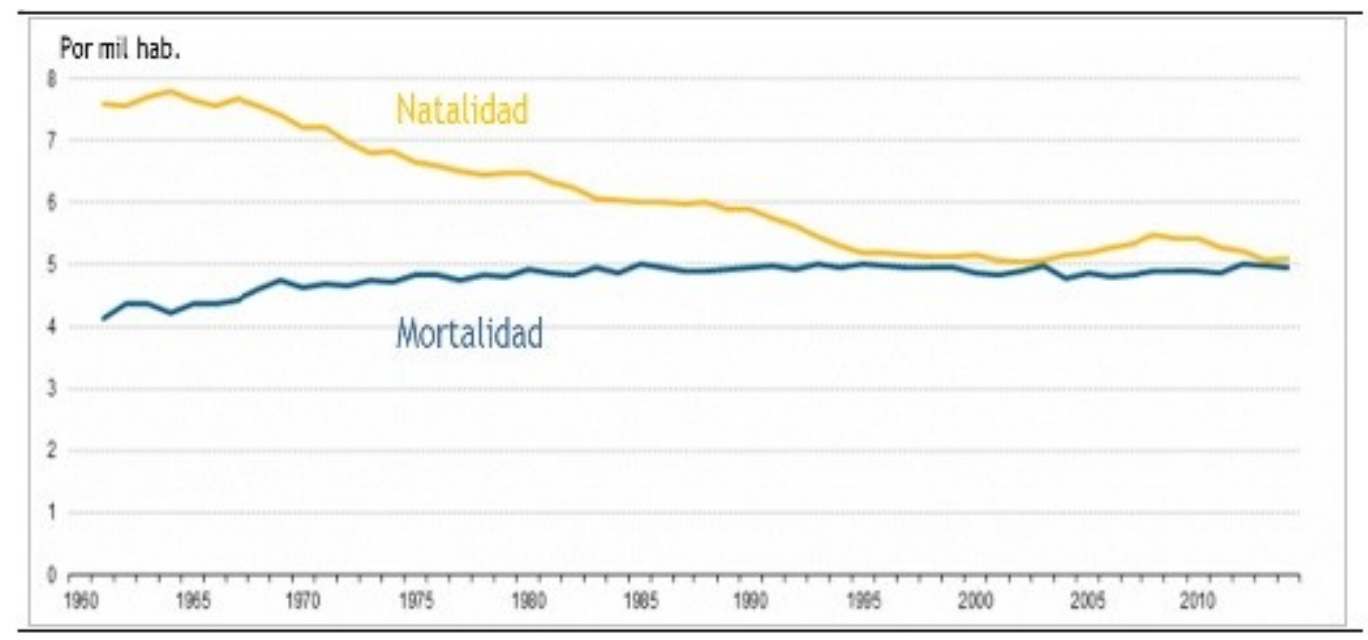

Fuentes: Eurostat, 2016.

En la Unión Europea hay 500 millones de habitantes y esta población está estabilizada con tasas de mortalidad y de natalidad que se neutralizan (figura 11). La población en Europa no aumenta en su saldo natural. Con una población vieja, la tasa de mortalidad va a aumentar en los próximos años, sin esperanza de que aumente la tasa de natalidad. Este fenómeno es más importante en el este de Europa (Alemania, Italia, Grecia y los antiguos países del bloque soviético) que en la Europa del Oeste (Irlanda, Francia, sur de España)

Por suerte, en 2014, había 6\% de personas extranjeras que vivían en Europa, 34 millones, pero un 40\% son de otros países europeos y únicamente 20 millones son de un país realmente extranjero. Cada año hay 3,8 millones de personas que llegan a Europa y 2,8 millones que se van (figura 12). 
Figura 12 - Europeos y extranjeros en Europa en $2015^{\underline{3}}$

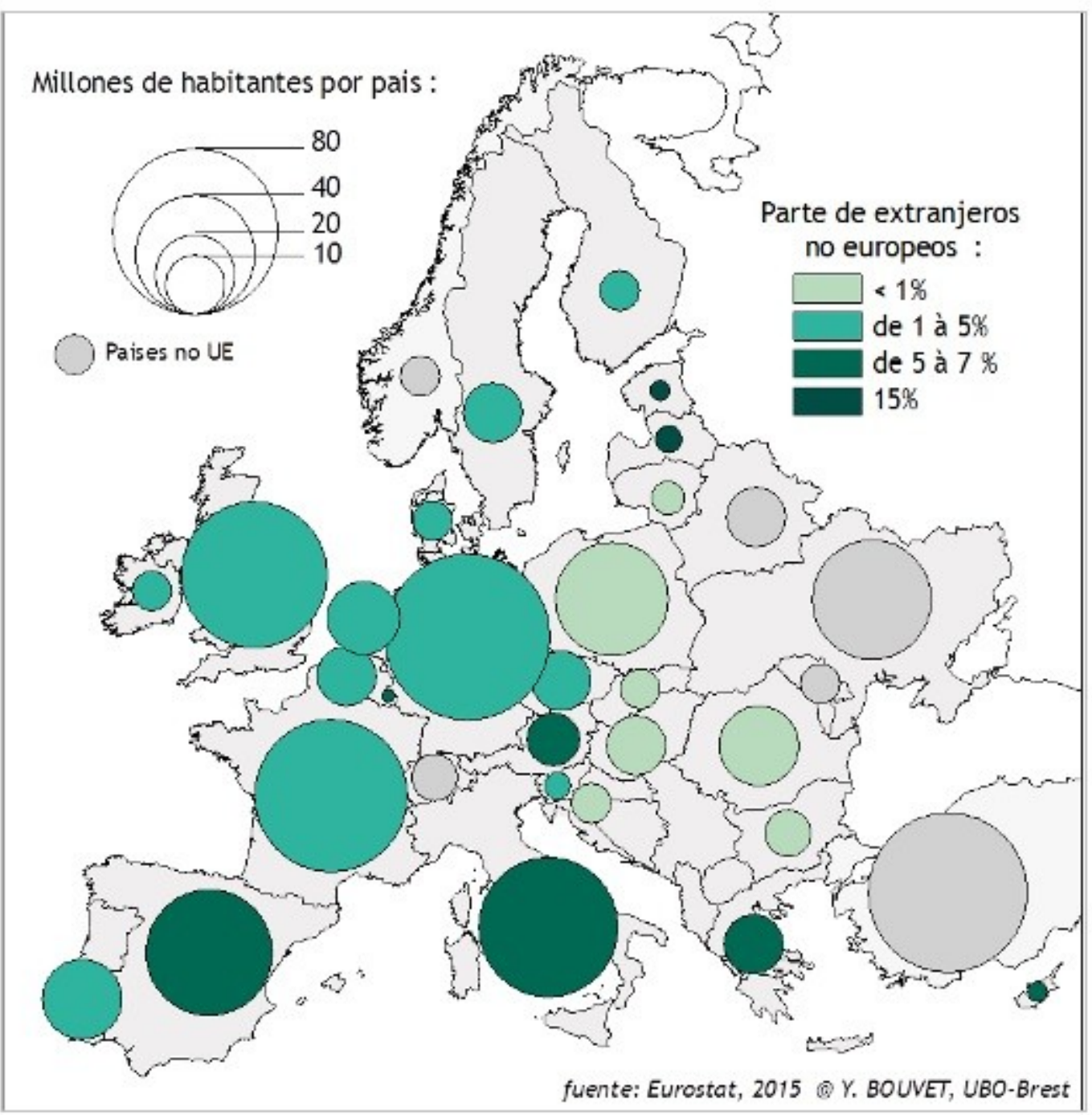

Los datos provienen de Eurostat, la oficina de estadísticas de la Unión Europea, que posee información disponible sobre todas las poblaciones que viven en Europa con datos sobre edades, sexo, nivel de diploma, situación de familia. Así sabemos que las personas que llegan a Europa son más jóvenes que los europeos, con buen nivel de educación, pueden trabajar y también tener hijos para dar nuevos habitantes a la región.

El contexto mundial presentaun aumento espectacular: en 2005 había en el mundo 20 millones de refugiados, en 2015 hay 53 millones. Sin embargo, no estamos seguros sobre los datos,dado que sólo están los del Alto Comisario de los Refugiados de ONU. Hay muchos conflictos en el Mundo, 8 en África, 3 en Medio Oriente (Yemen, Irak, Siria), en Europa (Ucrania), en Asia (Pakistán, Afganistán), y guerras civiles (Colombia, Turquía) (figura 13),por lo que en 2015, las tres primeras poblaciones de refugiados eran:

- Siria, con 12 millones de personas sobre un total de 23 millones, la mitad de la población.

- En segundo lugar estaban los colombianos con 6 millones de personas (muchos para Colombia)

- La población de Irak es la tercera con 4,1 millones de refugiados (un tercio de la población quedó adentro, dos tercios afuera).

Están los países vecinos, que tienen el más importante número de refugiados: 
- Pakistán por los refugiados de Afganistán

- Irán por Irak

- Líbano y Jordania por Siria

Figura 13 De donde son los refugiados

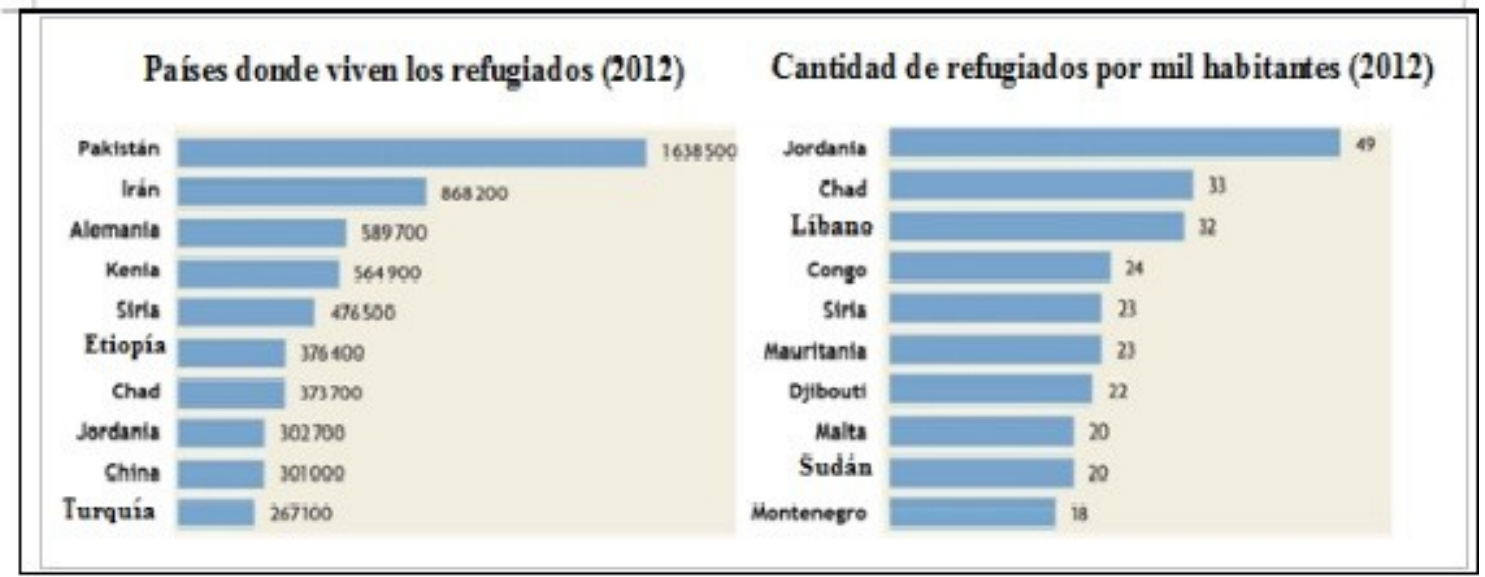

Fuente: UNHCR, 2013) $)^{4}$

Europa no es el primer destino de los refugiados de Medio Oriente, de África o de Asia,pero sufre una crisis importante debido a las llegadas de refugiados, con casi 1 millón de personas en 2015, y por lo menos la misma cantidad en 2016, aunque tal vez más. Es una dificultad porque los refugiados arriban a un espacio político frágil, con dudas sobre qué es Europa, con países conservadores, católicos, partidos populistas, con tasas de desempleo muy fuertes (España, Italia, Francia, Europa del Este), en crisis financiera (Grecia, Italia), y con el Brexit (Inglaterra).

Cada país tiene razones para recibir o no a los refugiados, y una de ellas no es la diferencia de cultura, sino el deseo de preservar la vida de los seres humanos.

Para concluir esta presentación, podemos decir que Francia es un país en donde la población aumenta de manera natural,pero es el producto de una mezcla que, desde hace dos siglos viene haciendo que se encuentre la población de "origen” y las poblaciones inmigrantes mayoritariamente provenientes de Europa y de los países vecinos del Mediterráneo. La posición particular de Francia entre el Atlántico y el Mediterráneo, y entre Europa del norte y del este, la convierte en un puente entre los países europeos, y África y Medio Oriente,lo cual puede resultar una oportunidad paraun país viejo pero no para los países del sur que están perdiendo la parte más progresista de sus poblaciones.

\section{Notas}

1 Texto revisado, corregido y adaptado por Héctor Dupuy.

$\underline{2}$ Para tener datos sobre la poblaciónde Francia, utilizar el sitio de INSEE (Institutnational de la statistique et des étudeséconomiques): $\underline{\text { http://www.insee.fr/fr/statistiques/ }}$ 
$\underline{3}$ Para tener datos de Europa, utilizar el sitio de EUROSTAT : http://ec.europa.eu/eurostat/web/populationdemography-migration-projections/population-data/database

$4 \underline{\text { http://www.unhcr.org/51bacb0f9.html }}$

\section{Bibliografía}

Adveev,A.et al.(2011). Populations et tendances démographiques des pays européens (19802010).Population-F, INED, 66(1), 9-133.

Eurostat(2015). Citoyens étrangers résidant dans les états membres de l’UE, communiqué de presse 230/2015, 18 décembre, 4 pages.

Insee Première (2014).Les immigrés récemment arrivés en France, une immigration de plus en plus européenne, $\mathrm{n}^{\circ} 1524$, novembre, 4 pages.

Mazuy, M.et al.(2015). L'évolution démographique récente de la France et ses tendances depuis 70 ans. Population-F, INED, 70(3), 417- 486.

http://www.unhcr.org

http://ec.europa.eu/eurostat/web/population-demography-migration-projections/population-data/database http://www.insee.fr/fr/statistiques/ 